\title{
A Parallel Solver for Anisotropic Cardiac Models
}

\author{
P Colli-Franzone ${ }^{1,2}$, L F Pavarino $^{3}$, B Taccardi $^{4}$ \\ ${ }^{1}$ Dipartimento di Matematica, Universitá di Pavia, Italy \\ ${ }^{2}$ Istituto di Matematica Applicata e Tecnologie Informatiche, Pavia, Italy \\ ${ }^{3}$ Dipartimento di Matematica, Universitá di Milano, Milano, Italy \\ ${ }^{4}$ Cardiovascular Research and Training Institute, University of Utah Salt Lake City, Utah
}

\begin{abstract}
A parallel solver for numerical simulations of a full cardiac cycle in three dimensional domains, based on the anisotropic Monodomain and Bidomain models, is presented. The solver employs structured isoparametric trilinear finite elements in space and a semi-implicit adaptive method in time. Parallelization and portability are based on the PETSc parallel library. Largescale parallel computations have been run, simulating anisotropic dispersion of the action potential duration.
\end{abstract}

\section{Introduction}

Knowledge of the rules that govern the full cardiac cycle is a prerequisite for understanding and interpreting abnormal sequences that occur in conduction disturbances. The excitation and recovery phases are influenced by the direction of the myocardial fibers and by the anisotropic conductivity of the intra and extracellular media. While the former phase has been examined in considerable detail both experimentally and numerically (see $[1,2,3]$ ), much less is known concerning the latter (see $[4,5,6$, 7]). During a normal heartbeat, the time course of the ventricular transmembrane potential displays mainly three phases having different time and space scales. At first in the excitation phase, a moving layer associated with the upstroke of the action potential sweeps the entire cardiac domain. Subsequently, small spatial and temporal potential variations are observed in the long plateau phase and finally smooth changes, in space and time, are associated with the repolarization phase. To tackle the high computational costs involved in large scale simulations of a full cardiac cycle in a three dimensional domain, adaptive and parallel tools are required.

\section{Mathematical models}

The Bidomain model. In the Bidomain approach, the anisotropy of the two averaged continuous media, the intra and the extracellular medium, are characterized by the conductivity tensors $D_{i}(\mathbf{x})$ and $D_{e}(\mathbf{x})$ related to the arrangement of the cardiac fibers which rotate counterclockwise from the epicardium to the endocardium, (see [8]). Moreover, from [9], the cardiac tissue has a laminar organization and may be conceived of as a set of muscle sheets running radially from epi to endocardium. Therefore, at any point $\mathbf{x}$, it is possible to identify a triplet of orthonormal principal axes $\mathbf{a}_{l}(\mathbf{x}), \mathbf{a}_{t}(\mathbf{x}), \mathbf{a}_{n}(\mathbf{x})$, with $\mathbf{a}_{l}(\mathbf{x})$ parallel to the local fiber direction, $\mathbf{a}_{t}(\mathbf{x})$ and $\mathbf{a}_{n}(\mathbf{x})$ tangent and orthogonal to the radial laminae respectively and both being transversal to the fiber axis. Denoting by $\sigma_{l}^{i, e}, \sigma_{t}^{i, e}, \sigma_{n}^{i, e}$ the conductivity coefficients measured along the corresponding directions, then the conductivity tensors $D_{i}(\mathbf{x})$ and $D_{e}(\mathbf{x})$ related to orthotropic anisotropy of the media are given by:

$$
D_{i, e}=\sigma_{l}^{i, e} \mathbf{a}_{l} \mathbf{a}_{l}^{T}+\sigma_{t}^{i, e} \mathbf{a}_{t} \mathbf{a}_{t}^{T}+\sigma_{n}^{i, e} \mathbf{a}_{n} \mathbf{a}_{n}^{T}
$$

while for axially isotropic media, i.e. $\sigma_{n}^{i, e}=\sigma_{t}^{i, e}$, we have

$$
D_{i, e}=\sigma_{t}{ }^{i, e} I+\left(\sigma_{l}{ }^{i, e}-\sigma_{t}{ }^{i, e}\right) \mathbf{a}_{l} \mathbf{a}_{l}^{T} .
$$

The intra and extracellular electric potentials $u_{i}, u_{e}$ in the Bidomain model are described by a reaction-diffusion system coupled with a system of ODEs for ionic gating variables $w$. Given the applied currents per unit volume $I_{a p p}^{i, e}$, satisfying the compatibility condition $\int_{H} I_{a p p}^{i} \mathrm{dx}=$ $\int_{\mathrm{H}} \mathrm{I}_{\mathrm{app}}^{\mathrm{e}} \mathrm{dx}$, the initial conditions $v_{0}, w_{0}$, then, for an insulated cardiac domain $H, u_{i}, u_{e}, w$ satisfy the system:

$$
\left\{\begin{array}{c}
c_{m} \partial_{t} v-\operatorname{div}\left(D_{i} \nabla u_{i}\right)+I_{i o n}(v, w)=I_{a p p}^{i} \\
-c_{m} \partial_{t} v-\operatorname{div}\left(D_{e} \nabla u_{e}\right)-I_{i o n}(v, w)=-I_{a p p}^{e} \\
\partial_{t} w-R(v, w)=0, \quad v(t)=u_{i}(t)-u_{e}(t) \\
\mathbf{n}^{T} D_{i} \nabla u_{i}=0, \mathbf{n}^{T} D_{e} \nabla u_{e}=0 \\
v(\mathbf{x}, 0)=v_{0}(\mathbf{x}), w(\mathbf{x}, 0)=w_{0}(\mathbf{x}),
\end{array}\right.
$$

where $\partial_{t}=\partial / \partial t, c_{m}=\chi * C_{m}, I_{i o n}=\chi * i_{\text {ion }}$, with $\chi$ the ratio of membrane area per tissue volume, $C_{m}$ the surface capacitance and $i_{i o n}$ the ionic current of the membrane per unit area. The system uniquely determines $v$, while the potentials $u_{i}$ and $u_{e}$ are defined only up to a same additive time-dependent constant related to the reference potential, chosen to be the average extracellular potential in the cardiac volume by imposing $\int_{H} u_{e} \mathrm{dx}=0$.

The Monodomain model. Assuming equal anisotropy ratio of the two media, i.e. $D_{i}=\lambda D_{e}$ with $\lambda$ constant, 
then the Bidomain system reduces to the anisotropic Monodomain model consisting in a parabolic reactiondiffusion equation for the transmembrane potential $v$ coupled with a system of ODEs for gating variables:

$$
\left\{\begin{array}{l}
c_{m} \partial_{t} v-\operatorname{div}\left(D_{m}(\mathbf{x}) \nabla v\right)+I_{i o n}(v, w)=I_{a p p}^{m}, \\
\partial_{t} w-R(v, w)=0, w(\mathbf{x}, 0)=w_{0}(\mathbf{x}), \\
\mathbf{n}^{T} D_{m} \nabla v=0, \quad v(\mathbf{x}, 0)=v_{0}(\mathbf{x}),
\end{array}\right.
$$

where $D_{m}=\sigma_{l} \mathbf{a}_{l} \mathbf{a}_{l}^{T}+\sigma_{t} \mathbf{a}_{t} \mathbf{a}_{t}^{T}+\sigma_{n} \mathbf{a}_{n} \mathbf{a}_{n}^{T}$, with $\sigma_{l, t, n}=$ $\lambda \sigma_{l, t, n}^{i} /(1+\lambda)$ and $I_{a p p}^{m}=\left(\lambda I_{a p p}^{i}+I_{a p p}^{e}\right) /(1+\lambda)$.

\section{Numerical solver}

Finite element space discretization. The computer solver for the simulation of the mono- and bi-domain models is applied to cardiac tissue volume $H$ assuming that the domain is discretized by a structured grid made up of hexahedral isoparametric $Q_{1}$ elements and we denote by $V_{h}$ the associated finite element space. A semidiscrete problem is obtained by applying a standard Galerkin procedure and choosing a finite element basis $\left\{\phi_{i}\right\}$ for $V_{h}$. We denote by $\mathrm{M}=\left\{\mathrm{m}_{\mathrm{rs}}=\int_{\mathrm{H}} \varphi_{\mathrm{r}} \varphi_{\mathrm{s}} \mathrm{dx}\right\}$ the symmetric mass, by $\mathrm{A}_{\mathrm{m}, \mathrm{i}, \mathrm{e}}=\left\{\mathrm{a}_{\mathrm{rs}}^{\mathrm{m}, \mathrm{i}, \mathrm{e}}=\int_{\mathrm{H}}\left(\nabla \varphi_{\mathrm{r}}\right)^{\mathrm{T}} \mathrm{D}_{\mathrm{m}, \mathrm{i}, \mathrm{e}} \nabla \varphi_{\mathrm{s}} \mathrm{dx}\right\}$ the symmetric stiffness matrices and by $\mathrm{I}_{\mathrm{ion}}^{\mathrm{h}}, \mathrm{I}_{\mathrm{app}}^{(\mathrm{m}, \mathrm{i}, \mathrm{e}), \mathrm{h}}$ the finite element interpolants of $I_{i o n}$ and $I_{a p p}^{m, i, e}$, respectively.

Semi-implicit time discretizations. The time discretization is performed by a semi-implicit method using for the diffusion term the implicit Euler method, while the nonlinear reaction term $I_{i o n}$ is treated explicitly. The use of an implicit treatment of the diffusion terms appearing in the Mono- or Bi-domain models is essential to allow an adaptive change of the time step according to the stiffness of the various phases of the heartbeat. The ODE system for the gating variables is discretized by the semi-implicit Euler method; in this way we decouple the gating variables by solving the gating system first (given the potential $\mathbf{v}^{\mathbf{n}}$ at the previous time-step)

$$
\left(\mathbf{w}^{\mathbf{n}+\mathbf{1}}-\mathbf{w}^{\mathbf{n}}\right) / \Delta t=R\left(\mathbf{v}^{\mathbf{n}}, \mathbf{w}^{\mathbf{n}+\mathbf{1}}\right)
$$

and then solving for $\mathbf{u}_{\mathbf{i}}^{\mathbf{n}+\mathbf{1}}, \mathbf{u}_{\mathbf{e}}^{\mathbf{n}+\mathbf{1}}$ in the Bidomain case

$$
\left(\frac{c_{m}}{\Delta t}\left[\begin{array}{cc}
\mathrm{M} & -\mathrm{M} \\
-\mathrm{M} & \mathrm{M}
\end{array}\right]+\left[\begin{array}{cc}
\mathrm{A}_{\mathrm{i}} & 0 \\
0 & \mathrm{~A}_{\mathrm{e}}
\end{array}\right]\right)\left(\begin{array}{l}
\mathbf{u}_{\mathbf{i}}^{\mathbf{n}+\mathbf{1}} \\
\mathbf{u}_{\mathbf{e}}^{\mathbf{n}+\mathbf{1}}
\end{array}\right)=
$$

$\frac{c_{m}}{\Delta t}\left(\begin{array}{c}\mathrm{M}\left(\mathbf{u}_{\mathbf{i}}^{\mathbf{n}}-\mathbf{u}_{\mathbf{e}}^{\mathbf{n}}\right) \\ \mathrm{M}\left[-\mathbf{u}_{\mathbf{i}}^{\mathbf{n}}+\mathbf{u}_{\mathbf{e}}^{\mathbf{n}}\right]\end{array}\right)+\left(\begin{array}{c}\mathrm{M}\left[-\mathrm{I}_{\mathrm{ion}}^{\mathrm{h}}\left(\mathbf{v}^{\mathbf{n}}, \mathbf{w}^{\mathbf{n}+\mathbf{1}}\right)+\mathrm{I}_{\mathrm{app}}^{\mathrm{i}, \mathrm{h}}\right] \\ \mathrm{M}\left[\mathrm{I}_{\mathrm{ion}}^{\mathrm{h}}\left(\mathbf{v}^{\mathbf{n}}, \mathbf{w}^{\mathbf{n}+\mathbf{1}}\right)-\mathrm{I}_{\mathrm{app}}^{\mathrm{e}, \mathrm{h}}\right]\end{array}\right)$ where $\mathbf{v}^{\mathbf{n}}=\mathbf{u}_{\mathbf{i}}^{\mathbf{n}}-\mathbf{u}_{\mathbf{e}}^{\mathbf{n}}$; as in the continuous model, $\mathbf{v}^{\mathbf{n}}$ is uniquely determined, while $\mathbf{u}_{\mathbf{i}}^{\mathbf{n}}$ and $\mathbf{u}_{\mathbf{e}}^{\mathbf{n}}$ are determined only up to the same additive time-dependent constant chosen by imposing the condition $\mathbf{M} \mathbf{u}_{\mathbf{e}}^{\mathbf{n}}=\mathbf{0}$.

In the Monodomain case we have to solve for $\mathbf{v}^{n+1}$

$$
\left(\frac{c_{m}}{\Delta t} \mathrm{M}+\mathrm{A}\right) \mathbf{v}^{\mathbf{n}+\mathbf{1}}=\frac{\mathbf{c}_{\mathbf{m}}}{\boldsymbol{\Delta} \mathbf{t}} \mathrm{Mv}^{\mathbf{n}}-\mathrm{MI}_{\mathrm{ion}}^{\mathrm{h}}\left(\mathbf{v}^{\mathbf{n}}, \mathbf{w}^{\mathbf{n}+\mathbf{1}}\right)+\mathrm{MI}_{\mathrm{app}}^{\mathrm{m}, \mathrm{h}}
$$

The solution of the discrete model is computationally expensive because of the involvement of different space and time scales. Due to the high computational costs, adaptive and parallel tools are then required in order to successfully complete large-scale simulations. While both tools can in principle be applied to both space and time, we have chosen to use adaptive methods in time and parallel solvers in space. We employed an adaptive time-stepping strategy based on controlling the transmembrane potential variation $\Delta v=\max \left(\mathbf{v}^{\mathbf{n}+\mathbf{1}}-\mathbf{v}^{\mathbf{n}}\right)$ at each time-step, see [10]. The dynamics of $S$ gating variables are described by equations of the form $\partial_{t} w_{j}=R_{j}\left(v, w_{j}\right)=\left(w_{j \infty}(v)-w_{j}\right) / \tau_{j}(v)$ for $j=1, \ldots, S$; in order to guarantee a control on the variation of $\mathbf{w}_{j}$ too [11], given $\mathbf{v}^{n}$, due to the linearity in $\mathbf{w}_{j}$, the equation is integrated exactly. In this paper, we consider the Luo-Rudy phase I (LR1) model (see [10]), since it is one of the complex gating systems mostly used in recents 3D simulations; in this model the calcium ionic concentration is updated using the explicit Euler method. Numerical quadrature with a 3D trapezoidal rule is used to compute the integrals, therefore the mass matrix $M$ is lumped to diagonal form. In our implementation, we have actually reordered the unknowns writing for every node the $\mathbf{u}_{\mathbf{i}}$ and $\mathbf{u}_{\mathbf{e}}$ components consecutively, so as to minimize bandwidth of the stiffness matrix. The linear system at each time step in the discrete problems is solved iteratively by the preconditioned conjugate gradient (PCG) method, using as initial guess the solution at the previous time step. Parallelization and portability are realized using the PETSc parallel library [12] and a preconditioned conjugate gradient solver at each time step with block Jacobi preconditioner and ILU(0) on each block.

\section{Results}

We have performed several numerical experiments in three dimensions on parallel architectures, with both the Monodomain and the Bidomain models. The parallel machines employed are an IBM SP RS/6000 Power4 with 512 processors Power 4 - $1300 \mathrm{MHz}$, grouped into 16 nodes of 32 processors and 16 GB RAM each (www.cineca.it), and an HP SuperDome 64000 with 64 processors PA8700 - $750 \mathrm{MHz}$ and $64 \mathrm{~GB}$ RAM (www.cilea.it). The domains considered are either cartesian slabs or truncated ellipsoids modeling portions of the left ventricle. In both cases a structured grid of $\left(n_{i} \cdot n_{j} \cdot n_{k}\right)$ hexahedral isoparametric $Q_{1}$ elements was used. In the numerical tests, we have used the following parameters: $\chi=$ $10^{3} \mathrm{~cm}^{-1}, C_{m}=10^{-3} \mathrm{mF} / \mathrm{cm}^{2},\left\{\sigma_{l}^{e}, \sigma_{l}^{i}, \sigma_{t}^{e}, \sigma_{t}^{i}\right\}=$ $\{2,3,1.35,0.315\} m \Omega^{-1} \mathrm{~cm}^{-1}$ and $\sigma_{n}^{e}=\sigma_{t}^{e} / 2, \sigma_{n}^{i}=$ $\sigma_{t}^{i} / 10$ in the orthotropic case. In the Monodomain model $D_{m}$ is defined by setting $\sigma_{l, t, n}=\sigma_{l, t, n}^{i} \sigma_{l, t, n}^{e} /\left(\sigma_{l, t, n}^{i}+\right.$ $\left.\sigma_{l, t, n}^{e}\right)$ in order to obtain, at first order, the same conduction velocity along and across fibers as in the Bidomain model. As in [2], the fibers rotate intramurally linearly, proceeding counterclockwise from epicardium to endocardium, for a total amount of $120^{\circ}$ and of $90^{\circ}$ in the ellipsoidal and in 
Table 1. Monodomain and Bidomain with LR1 model. $\mathrm{AT}=$ assembling time, $\mathrm{IN}=$ average number of $\mathrm{PCG}$ iterations per time-step, TS= average time per time-step

\begin{tabular}{|ccccc|}
\hline \multicolumn{5}{c|}{ Monodomain with LR1 model } \\
\# proc. & mesh & AT & IN & TS \\
\hline 8 & $150 \cdot 150 \cdot 100$ & $7.7 \mathrm{~s}$ & 4 & $2.7 \mathrm{~s}$ \\
16 & $300 \cdot 150 \cdot 100$ & $8.5 \mathrm{~s}$ & 4 & $3 \mathrm{~s}$ \\
32 & $300 \cdot 300 \cdot 100$ & $9.1 \mathrm{~s}$ & 5 & $3.6 \mathrm{~s}$ \\
64 & $600 \cdot 300 \cdot 100$ & $9.2 \mathrm{~s}$ & 5 & $3.6 \mathrm{~s}$ \\
128 & $600 \cdot 600 \cdot 100$ & $10.6 \mathrm{~s}$ & 8 & $5.1 \mathrm{~s}$ \\
\hline \hline \multicolumn{6}{c|}{ Bidomain with LR1 model } \\
\# proc. & mesh & AT & IN & TS \\
\hline 8 & $100 \cdot 100 \cdot 70$ & $12.9 \mathrm{~s}$ & 98 & $40.2 \mathrm{~s}$ \\
16 & $200 \cdot 100 \cdot 70$ & $13.3 \mathrm{~s}$ & 127 & $55.5 \mathrm{~s}$ \\
32 & $200 \cdot 200 \cdot 70$ & $15.7 \mathrm{~s}$ & 148 & $72 \mathrm{~s}$ \\
64 & $400 \cdot 200 \cdot 70$ & $16.2 \mathrm{~s}$ & 176 & $91.9 \mathrm{~s}$ \\
128 & $400 \cdot 400 \cdot 70$ & $18.4 \mathrm{~s}$ & 244 & $129.7 \mathrm{~s}$ \\
\hline
\end{tabular}

the slab geometry respectively. The initial conditions are $\left(\mathbf{u}_{i}^{0}, \mathbf{u}_{e}^{0}\right)=(-84,0) \mathrm{mV}$, so that $\mathbf{v}^{0}=-84 \mathrm{mV}$ and we apply a stimulus of $250 \mathrm{~mA}$ for $1 \mathrm{msec}$ on a small area (3 or 5 mesh points in each direction). Other than potentials and gating variables, at each time-step, we compute also the activation (ACTI) and the repolarization (REPO) times, defined as the times when the action potential (AP) crosses $-60 \mathrm{mV}$ during the upstroke and the downstroke respectively. In the LR 1 model the slow inward current was scaled by a factor $2 / 3$, yielding an action potential duration (APD) of about $265 \mathrm{msec}$. In order to evaluate the performance of the parallel solver, we have considered the Mono and Bi-domain models with LR1 gating, simulating the initial depolarization of some ellipsoidal sections. The sections are chosen in increasing sizes so as to keep constant the number of mesh points per subdomain (processor). The domain varies from the smaller section with 8 subdomains to half ventricle with 128 subdomains. The computing platform is the IBM SP4. The results, reported in Table 1, show for the Monodomain model that the algorithm seems practically scalable, and even for 128 processors, the number of PCG iterations grows to just 8; the results for the Bidomain model indicate that while the assembling time remains reasonable (under $20 \mathrm{sec}$ ), the average number of PCG iterations per time-step and the average time per time-step are now much larger. This is not only due to the doubling of the unknowns, but can be attributed to the limits of the one-level preconditioner and to the severe illconditioning of the Bidomain iteration matrix.

Full cardiac cycle. In order to study the influence of fiber rotational anisotropy on the repolarization sequence and on the dispersion of APD, we simulate a complete cardiac cycle in a slab of cardiac tissue. The fibers rotate intramurally linearly with depth for a total amount of $90^{\circ}$, i.e. $\mathbf{a}_{l}(\mathbf{x})=\mathbf{e}_{x} \cos \alpha(r)+\mathbf{e}_{y} \sin \alpha(r), \alpha(r)=$

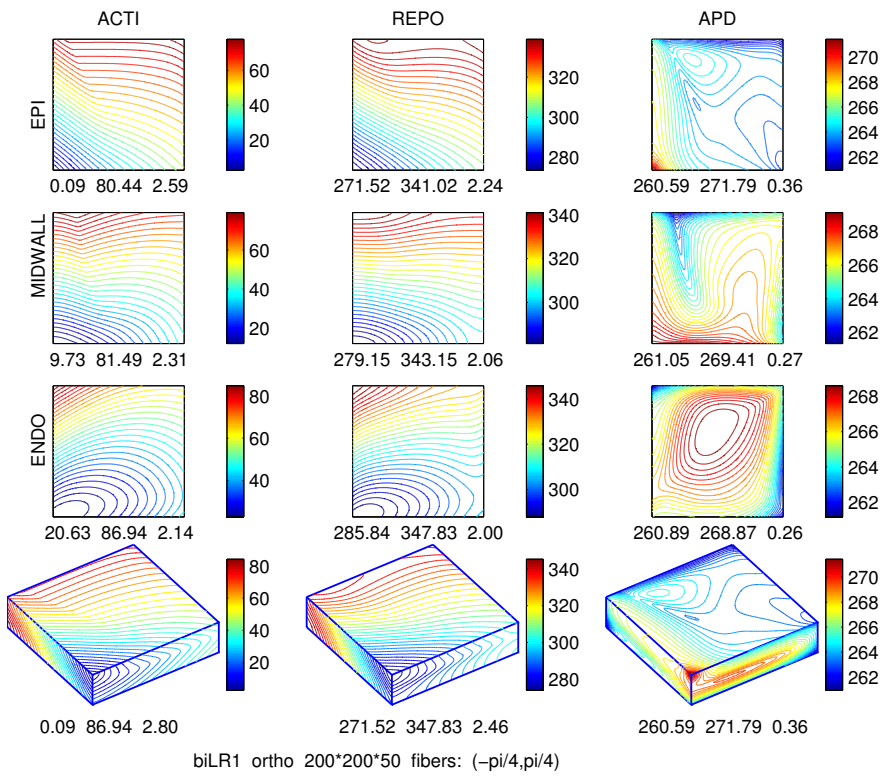

Figure 1. Orthotropic Bidomain slab: $2 \times 2 \times 0.5 \mathrm{~cm}^{3}$. Vertex stimulation in a slab without heterogeneity of the cellular membrane properties. Isochrone lines of the depolarization time (first column ACTI ), repolarization time ( second column REPO ) and action potential duration (third column APD); reported below each panels are the maximum, minimum and step in msec of the displayed map.

$\pi(1-2 r) / 4, r \in[0,1]$; on the upper-epicardial (lowerendocardial) face of the slab the fiber direction is $-45^{\circ}$ $\left(45^{\circ}\right)$. In the orthotropic anisotropy case, we choose $\mathbf{a}_{n}=$ $\mathbf{e}_{x} \sin \alpha(r)-\mathbf{e}_{y} \cos \alpha(r)$ and $\mathbf{a}_{t}=\mathbf{e}_{z}$. In order to emphasized the effects of the fiber rotational anisotropy, we apply a stimulus at an epicardial vertex of the slab in which wave front propagation is mainly across the epicardial fiber. Since the excitation of the entire slab requires about 80 msec, the time interval for simulating the cardiac cycle is on the order of $360 \mathrm{msec}$. The adaptive time-stepping algorithm automatically adapts, in an efficient way, the time step size in the three main different phases of the heart beat, while the number of PCG iterations of the linear solver change considerably, increasing to a maximum of about 250 iterations in the depolarization phase, indicating that our preconditioner and/or initial guess are not yet satisfactory. Simulation of a full cardiac cycle on a slab of size $2 \times 2 \times 0.5 \mathrm{~cm}^{3}$, discretized with a fine mesh $200 \times 200 \times$ 50 , using 25 processors of the HP SuperDome machine, took about 6.4 days for the the Bidomain model and about 5 hours for the Monodomain model. We compared the performance of the two computer platforms mentioned above by simulating the Monodomain model on a slab with dimensions $4 \times 4 \times 0.5 \mathrm{~cm}^{3}$ and mesh $400 \times 400 \times 50$ : the HP SuperDome machine with 32 processors took about 20 hours and the IBM SP4 machine with 64 processors took about 2.5 hours. The isochrone lines of the ACTI, REPO 


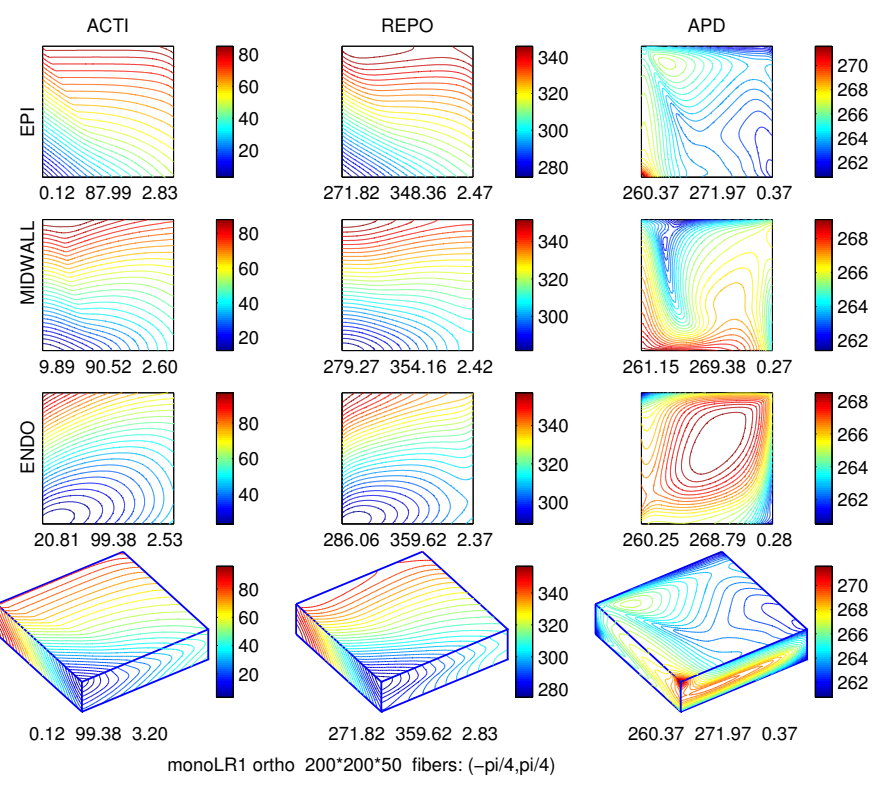

Figure 2. Orthotropic Monodomain slab: $2 \times 2 \times 0.5 \mathrm{~cm}^{3}$. Same format as in Fig.1

and APD are displayed in Fig. 1 for a Bidomain model with orthotropic anisotropy. Repolarization wave fronts exhibit a somewhat smoother shape and faster propagation compared with the excitation sequence as shown by the isochronal lines on the epi, midwall and endocardial layers. We underline that the APD patterns show a spatial dispersion in spite of the homogeneity of the individual cellular membrane properties assumed. Moreover the simulation of the orthotropic Monodomain model, (see Fig. 2), exhibits the same qualitative features of the ACTI, REPO and APD patterns as displayed in the Bidomain model. Therefore the anisotropic feature of the APD dispersion can be examined, at a qualitative level, simulating the Monodomain model thus avoiding the higher cost required, at present, by the Bidomain simulations. In order to reduce the influence of the boundaries, we have also considered an orthotropic Monodomain model on a larger slab having dimensions $4 \times 4 \times 0.5 \mathrm{~cm}^{3}$, see Fig. 3. The comparison between Figs. 2 and 3 shows that the spatial features of the dispersion of the APD are not only due to boundary effects but can be partly attributed to the electrotonic modulation related to the fiber rotational anisotropy. We have also performed simulations in axially isotropic media obtaining the same, but less emphasized, anisotropic features of the APD dispersion compared with the orthotropic case. Future work will address the influence of intramural heterogeneity of membrane ionic currents, allowing for differences between epicardial, $\mathrm{M}$ cells and endocardial cells.

\section{References}

[1] Taccardi B, et al. Effect of myocardial fiber direction on epicardial potentials. Circulation 1994;90:3076-3090.

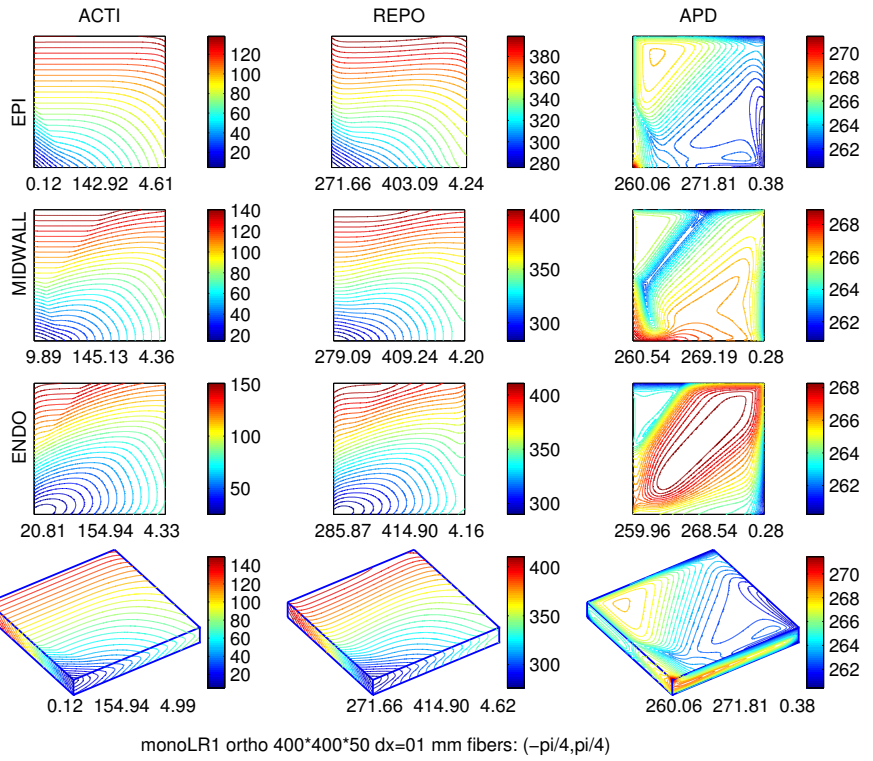

Figure 3. Orthotropic Monodomain slab: $4 \times 4 \times 0.5 \mathrm{~cm}^{3}$. Same format as in Fig.1.

[2] Colli Franzone P, et al. Anisotropic mechanisms for multiphasic unipolar electrograms. Simulation studies and experimental recordings. Ann Biomed Eng 2000;28:1-17.

[3] Muzikant A, et al. Region specific modeling of cardiac muscle: comparison of simulated and experimental potentials. Ann Biomed Eng 2003;30:867-883.

[4] Gotoh M, et al. Anisotropic repolarization in ventricular tissue. Am J Physiol 1987;41:107-113.

[5] Taccardi B, et al. Epicardial recovery sequences and excitation recovery intervals during paced beats. Role of epicardial architecture. PACE 1999;22 (4) part II:8-33.

[6] Efimov I, et al. Activation and repolarizationpatterns are governed by different structural characyteristics of ventricular myocardium: experimental study with voltagesensitive dyes and numerical simulations. J Cardivasc Electrophysiol 1996;7:512-530.

[7] Cates A, Pollard A. A model study of intramural dispersion of action potential duration in the canine pulmonary conus. Ann Biomed Eng 1998;26:567-576.

[8] Streeter D. Gross morphology and fiber geometry in the heart. In Berne R (ed.), Handbook of Physiology, vol. 1 The Heart. Baltimore: Williams \& Wilkins, 1979; 61-112.

[9] LeGrice I, et al. Laminar structure of the heart: ventricular myocyte arrangement and connective tissue architecture in the dog. Am J Physiol Heart Circ Physiol 1995;269 (38):H571-H582.

[10] Luo C, Rudy Y. A model of the ventricular cardiac action potential: depolarization, repolarization, and their interaction. Circ Res 1991;68 (6):1501-1526.

[11] Victorri A, et al. Numerical integration in the reconstruction of cardiac action potential using the Hodgkin-Huxley type models. Comp Biomed Res 1985;18:10-23.

[12] Balay S, et al. PETSc Users Manual. Tech. rep. anl-95/11 - revision 2.1.5 edition. Argonne National Laboratory, 2002. PETSc home page. http://www.mcs.anl.gov/petsc, 2001. 